A. Büttner K.-H. Rühle

\section{Lebensqualität vor und unter nCPAP}

\author{
Ein Vergleich mittels verschiedener Fragebogen - SWLS, MLDL und SF-36
}

\section{Quality of Life Before and During nCPAP. Comparison of Different \\ Questionnaires - SWLS, MLDL and SF-36}

\section{Zusammenfassung}

Das obstruktive Schlafapnoe-Syndrom (OSAS) wird von einer Beeinträchtigung der Tagesbefindlichkeit begleitet. Diese wiederum ist ein entscheidendes Kriterium der Lebensqualität. Fragebögen dienen als Instrumente zu ihrer Erfassung, Objektivierung und Verlaufsbeobachtung unter Therapie. Benutzt wurden u.a. Testverfahren wie Satisfaction With Life Scale SWLS: für globale Lebensqualität; Münchner Lebensqualitäts-Dimensionen-Liste MLDL und Short Form-36 des Health Survey SF-36: für bereichsspezifische Lebensqualität. Mit der vorliegenden Arbeit sollte nun geklärt werden, inwieweit und in welchen Bereichen sich die Lebensqualität von OSAS-Patienten sowohl nach Therapieeinleitung mit CPAP als auch unter Dauertherapie verbessert. Durch Vergleich der Effektstärken kann auch die Wertigkeit für die Therapiebeurteilung eingeschätzt werden. Zu diesem Zweck wurden 41 OSAS-Patienten und 40 gesunde Probanden die genannten Fragebögen vorgelegt, die sowohl Schlaf- als auch Lebensqualitätsparameter erfassten. Die Überprüfung der globalen Lebensqualität mittels Satisfaction With Life Scale (SWLS) ergab keinen signifikanten Unterschied zwischen den Probanden und den OSAS-Patienten. In der bereichspezifischen Lebensqualität wiesen 2 (von 4) Subskalen (Physische Zufriedenheit $\mathrm{p}=0,003$; Psychische Zufriedenheit $\mathrm{p}=0,001$ ) der Münchner Lebensqualitäts-Dimensionen-Liste (MLDL) Unterschiede zwischen den Gesunden und den untherapierten Patienten auf. Bei der Short Form-36 des Health Survey (SF-36) ergaben 7 (von 8) Subskalen signifikante Unterschiede zwischen diesen Gruppen (Ausnahme: Emotionale Rollenfunktion). Der Vergleich der Effektstärken ergab bei der Skala Vitalität im SF-36 nach > 6 Monaten den höchs-

\section{Abstract}

The obstructive sleep apnea syndrome is accompanied by an impairment of patients' condition during the day. The latter, in turn, is a decisive criterion quality of life. The instruments employed for data gathering, objectification and observation of the course of therapy are questionnaires. Questionnaires like Satisfaction With Life Scale SWLS: for global quality of life; Münchner Lebensqualitäts-Dimensionen-Liste ${ }^{1}$ MLDL and Short Form-36 of the Health Survey SF-36: for specific areas of life quality are often used. The purpose of this study is to clarify, to what extent and in which areas quality of life of OSAS patients improves both after initiation of therapy and under long-term therapy. By comparing effect size the value for effectiveness of therapy can be estimated. Thus, 41 OSAS patients and 40 controls were given an extensive set of tests addressing parameters of both sleep and quality of life. Testing of global quality of life with the Satisfaction With Life Scale (SWLS) did not reveal any significant difference between the controls and the OSAS patients. In specific araeas of life quality, 2 (of 4) subscales (Physical satisfaction: $p=0.003$; Psychological satisfaction: $p=0.001$ ) of the Münchner Lebensqualitäts-Dimensionen-Liste (MLDL) showed differences between the controls and the OSAS patients. For the Short Form-36 of the Health Survey (SF-36), 7 (of 8) subscales indicated significant differences between this groups (except for Emotional Role Function). The comparison of the effect size revealed the strongest effect in the subscale vitality of the SF-36 after more than 6

\footnotetext{
${ }^{1}$ Munich List of Life Quality Dimensions
} Universität Witten-Herdecke · Ambrocker Weg 60 · 58091 Hagen · E-mail: antja18b@yahoo.com 
ten Effekt $(0,93)$. Gute Effektstärken fanden sich in den Domänen Physische $(0,70)$ und Psychische Zufriedenheit der $\operatorname{MLDL}(0,56)$. Schlussfolgernd lässt sich sagen, dass die MLDL unter Berücksichtigung ihrer einfachen Durchführung und Auswertung in der klinischen Routine im Vergleich zur SF-36 das geeignetere Instrument zur Erfassung von verbesserter Lebensqualität durch eine nCPAP-Therapie darstellt. months (0.93). Good effect sizes were found in the domains Physical satisfaction (0.70) and Psychological satisfaction of the MLDL (0.56). In conclusion the MLDL is in consideration of its simple performance and evaluation compared with the SF-36 the better instrument in the clinical routine for the coverage of improved Quality of Life through a nCPAP therapy.

\section{Einleitung}

Seit einigen Jahren gewinnt der Begriff „Lebensqualität“ im schlafmedizinischen Bereich zunehmend an Bedeutung. Schlafstörungen beeinflussen nicht nur die Schlafqualität, sondern schränken die Betroffenen tagsüber teils erheblich ein $[2,13,14]$.

So führt insbesondere das obstruktive Schlafapnoe-Syndrom neben organischen Folgeerkrankungen zu einer Minderung der Tagesbefindlichkeit und der Lebensqualität sowie ihrer subjektiven Erlebbarkeit [2,10-12,17-19,21,23]. Eine Rolle spielt hierbei der Schweregrad der Erkrankung und die Dauer der bisher erfolgten Therapie [3,4,12,16,21]. Größere Untersuchungen bzgl. der Lebensqualität bei Patienten mit obstruktivem Schlafapnoesyndrom liegen insbesondere zur Münchner Lebensqualitäts-Dimensionen-Liste [1] und zur Short Form-36 des Health Survey [7] vor.

Aufgrund der bislang vorliegenden Befunde sollte die Untersuchung der Lebensqualität auch in der klinischen Routine verstärkt Eingang finden.

Ziel dieser Arbeit war es nun, die subjektiv erlebte globale sowie bereichsspezifische Lebensqualität bei OSAS-Patienten vor und nach nCPAP-Therapie zu untersuchen und die Effektstärke der unterschiedlichen Lebensqualitätsdomänen zu analysieren. Für diese Studie wurde daher ein Fragebogenpaket zusammengestellt, das sowohl klinische und demographische Daten (Diagnosebogen) als auch Parameter der Lebensqualität LQ (globale LQ: Satisfaction With Life Scale SWLS; bereichsspezifische LQ: Short Form-36 des Health Survey SF-36 und Münchner Lebensqualitäts-Dimensionen-Liste MLDL) erhob.

\section{Fragestellung}

Zur Erfassung der Lebensqualität werden verschiedene Fragebögen, die sich in ihrem Aufbau, ihrer Durchführung und Auswertung teilweise erheblich unterscheiden, verwendet. So erfasst die Satisfaction With Life Scale (SWLS) nur die globale Lebensqualität, die Münchner Lebensqualitäts-Dimensionen-Liste (MLDL) und Short Form-36 des Health Survey (SF-36) hingegen die allgemeine bereichsspezifische Lebensqualität. Die SF-36 ist gegenüber der MLDL jedoch sehr aufwändig, insbesondere bzgl. der Testzeit, der Verständlichkeit und der Auswertung [4]. Es sollte untersucht werden, welcher dieser Fragebogen für die klinische Routine praktikabel und gleichzeitig aussagekräftig genug ist.
Neben dem Vergleich der OSAS-Patienten mit Gesunden sollten daher durch einen Vergleich der Effektstärken diejenigen Bereiche (Domänen) der Lebensqualitäts-Fragebögen gefunden werden, welche die Verbesserung unter einer nCPAP-Therapie am besten beschreiben.

Fraglich ist in diesem Zusammenhang auch, ob kurzfristiges Abfragen sinnvoll ist oder ob sich die Lebensqualität der Betroffenen erst nach wochen- bzw. monatelanger Therapie verändert. Deshalb wurde in unserer Untersuchung die Lebensqualität nach 3 Tagen und mehr als 6 Monaten erfasst.

\section{Methodik}

\section{Studiendesign}

Die Untersuchung erfolgte an zwei Gruppen, wobei die OSAS-Patienten an drei Testzeitpunkten (vor Therapie, nach dreitägiger nCPAP-Therapie, nach mehr als sechs Monaten nCPAP) untersucht wurden, die Gruppe der gesunden Probanden an einem. Die OSAS-Patienten wurden anhand ihrer klinischen Diagnose (nach ICD-10) und die Kontrollgruppe aus der Allgemeinbevölkerung per Zufallsprinzip ausgewählt. Mittels der medizinischen Eingangsuntersuchung wurden Komorbiditäten bei den OSASPatienten ausgeschlossen, bei der gesunden Kontrollgruppe wurden mittels Fragebogen Vorerkrankungen erfasst. Komorbide Patienten sowie Kontrollen mit bekannten Vorerkrankungen wurden ausgeschlossen.

Die Probanden der Kontrollgruppe sollten möglichst das Altersspektrum der klinischen Gruppe abdecken. Alle Patienten wurden polysomnographisch untersucht, kannten ihre Diagnose und wurden bei ihrer Erstdiagnose bei einem AHI > 25/h ausgewählt.

Das Untersuchungsdesign sah sowohl den Vergleich der Lebensqualität dieser Patientengruppe im Therapieverlauf als auch den mit einer Kontrollgruppe vor. Zur Untersuchung des Therapieerfolgs wurde die Lebensqualität der OSAS-Patienten zu drei Testzeitpunkten erhoben (1: vor Therapie; 2: nach 3 Tagen CPAP; 3: > 6 Monaten CPAP [Zeitraum: 6-8 Monate]).

Die Datenerhebung erfolgte bei den 41 OSAS-Patienten (4 weiblich, 37 männlich) sowie den 40 Probanden (15 weiblich, 25 männlich) anhand einer Fragebogentestbatterie. Diese setzte sich aus vier Fragebögen zusammen, von denen der erste die klinisch-demographischen Daten erhob, der zweite die globale Lebensqualität mittels der Satisfaction With Life Scale SWLS $[15,22]$. Zwei weitere erfassten die bereichsspezifische Lebens- 
qualität mit der Münchner Lebensqualitäts-Dimensionen-Liste MLDL $[5,7,15,22]$ und der Short Form-36 des Health Survey SF-36 $[6,7,20]$.

\section{Messinstrumente \\ Demographische und klinische Daten}

Zur Erhebung der klinischen und demographischen Daten wurde ein Fragenbogen verwendet, der den klinischen Status des Krankheitsbildes sowie die Krankheitsdauer und die demographischen Angaben wie das Alter, das Geschlecht sowie die Schul- bzw. Ausbildung des Patienten erfasste.

\section{Erhebung der Lebensqualität}

In der Studie wurde sowohl die globale als auch die bereichsspezifische Lebensqualität erfasst.

Die Untersuchung der globalen Lebensqualität erfolgte mittels SWLS (Satisfaction With Life Scale), die der bereichsspezifischen Lebensqualität mittels der Fragebögen SF-36 (Short Form-36 des Health Survey) und MLDL (Münchner Lebensqualitäts-Dimensionen-Liste).

Satisfaction With Life Scale (SWLS)

Lebenszufriedenheit wird als das Ergebnis eines subjektiven Bewertungsprozesses definiert. Die SWLS-Konstruktion bestand ursprünglich aus drei Dimensionen (Zufriedenheit mit dem Leben, negative und positive Affekte).

Die in der Studie verwendete Version enthält 5 Items. Ihre Antwortmöglichkeiten sind siebenstufig, wobei eine hohe Punktzahl eine hohe Lebenszufriedenheit bedeutet.

Bei der 5-Item-Version erklärt der Hauptfaktor „Zufriedenheit“ $66 \%$ der Gesamtvarianz. Die innere Konsistenz liegt bei einem $\alpha$ von 0,87 , die Retest-Reliabilität (zwei-Monats-Abstand) beträgt $r_{\mathrm{tt}}=0,82[15,22]$.

Münchner Lebensqualitäts Dimensionen Liste (MLDL) Die MLDL dient zur Erfassung der multidimensionalen Lebensqualität. Sie erfasst 20 Dimensionen der Lebensqualität, die zu vier übergeordneten Kategorien (Physis, Psyche, Sozialleben, Alltagsleben) zusammengefasst worden sind.

Jede dieser Dimensionen wird zusätzlich anhand von vier Bewertungsaspekten (Zufriedenheit, Wichtigkeit, Veränderungswunsch, Veränderungsglauben) beurteilt. In der Studie wurde nur die Skala „Zufriedenheit“ verwendet.

Die 20 Dimensionen werden auf einer Skala von 0 bis 10 bewertet. Aus ihnen kann ein Gesamtwert über alle Bewertungsaspekte errechnet werden; ein hoher Gesamtwert entspricht einer hohen Lebensqualität.

Die ursprüngliche Normstichprobe bestand aus 144 Hypotonikern und 100 Gesunden. Die Skalenstruktur wurde nur z.T. in der Faktorenanalyse bestätigt. Die Subskalen besitzen teilweise hohe Interkorrelationen (zwischen $r=0,32$ und $r=0,55$ ). $62 \%$ der Gesamtvarianz können mit vier Faktoren erklärt werden.
Die innere Konsistenz der einzelnen Subskalen liegt zwischen $\alpha=0,64$ und $\alpha=0,80[5,15,22]$.

Short Form-36 des Health Survey (SF-36)

Die SF-36 erfasst verschiedene Bereiche der Lebensqualität. Sie untersucht die Auswirkungen von Krankheit und Behandlung auf den subjektiven Gesundheitszustand und die gesundheitsbezogene Lebensqualität.

Der Fragebogen besteht aus 8 Subskalen, die wesentliche Bereiche der Lebensqualität erfassen (Allgemeine Gesundheitswahrnehmung, Emotionale Rollenfunktion, Körperliche Funktionen, Körperliche Rollenfunktion, Psychisches Wohlbefinden, Schmerz, Soziale Funktionen, Vitalität).

Die Itemanzahl der verschiedenen Skalen schwankt zwischen 2 und 10, die Anzahl der Antwortmöglichkeiten liegt zwischen 2 und 6.

Zur Normierung liegen verschiedene Stichproben u.a. auch eine sehr umfangreiche deutsche vor: Die innere Konsistenz (Cronbach Alpha) der Subskalen liegt mehrheitlich über dem 0,07 Kriterium. Alters- bzw. Geschlechtsunterschiede konnten nicht gefunden werden $[6,20]$.

\section{Kontrollgruppe und OSAS-Patienten}

An der Untersuchung nahmen 41 Patienten mit obstruktiver Schlafapnoe (37 Männer, 4 Frauen) sowie 40 Gesunde (25 Männer, 15 Frauen) teil.

Die Diagnose obstruktives Schlafapnoe-Syndrom wurde anhand der Symptomatik und eines erhöhten AHI gestellt.

Voraussetzung zur Aufnahme in die Studie war eine Einwilligung zur Teilnahme (informed consent).

Um eine möglichst homogene Patientengruppe zu erhalten und verschiedene Störfaktoren (z.B. Alterseinflüsse) auszuschalten bzw. zu minimieren, wurden in der Studie folgende Ausschlusskriterien gewählt:

- Alter <65 Jahre

- keine weiteren Schlafstörungen

- keine organischen Erkrankungen

- keine psychische Beeinträchtigung

- Einnahme von Medikamenten jeder Art.

An der Studie nahmen insgesamt 81 Personen teil, 62 Männer (76,5\%) und 19 Frauen (23,5\%). Zu den weiteren demographischen und klinischen Daten siehe Tab.1.

Der Altersunterschied zwischen der Gruppe der gesunden Probanden und der der OSAS-Patienten ist in dieser Untersuchung nicht relevant, da für alle untersuchten Lebensqualitätsfragebögen keine Altersabhängigkeit gefunden wurde [5,6,15,20,22].

\section{Statistik}

Die Rohdaten wurden codiert, in eine Gesamtdatei übertragen und für das Statistikprogramm SPSS transformiert und ausgewertet. 
Tab.1 Demographische Daten der Gruppen

\begin{tabular}{|c|c|c|c|c|c|}
\hline Gruppe & Anzahl & $\begin{array}{l}\text { Altersmit- } \\
\text { telwerte }\end{array}$ & $A H I(/ h)$ & $\mathrm{O}_{2 \min }(\%)$ & BMI \\
\hline Gesunde & 40 & $42,1 \pm 12,1$ & & & $26,0 \pm 5,1$ \\
\hline $\begin{array}{l}\text { OSAS vor } \\
\text { Therapie }\end{array}$ & 41 & $53,6 \pm 13,0$ & $33,9 \pm 21,2$ & $81,3 \pm 6,7$ & $31,7 \pm 7,4$ \\
\hline $\begin{array}{l}\text { OSAS nach } \\
\text { Therapie- } \\
\text { einleitung }\end{array}$ & 41 & & $4,9 \pm 1,9$ & $86,4 \pm 10,7$ & \\
\hline $\begin{array}{l}\text { OSAS unter } \\
\text { Dauertherapie }\end{array}$ & 41 & & $1,1 \pm 3,7$ & $94,1 \pm 4,8$ & $32,1 \pm 6,3$ \\
\hline
\end{tabular}

$\mathrm{AHI}=$ Apnoe-Hypopnoe-Index; $\mathrm{O}_{2 \min }=$ minimale Sauerstoffsättigung Angabe des Alters, des AHIs und der $\mathrm{O}_{2}$-Sättigung in Mittelwerten $(\mathrm{x}) \pm$ Standardabweichung (S)

Die vier Gruppen wurden mit dem Kolmogorov-Smirnov-Test auf Normalverteilung überprüft. Dann wurde einerseits die Lebensqualität zwischen Gesunden und Schlafapnoepatienten, andererseits im Therapieverlauf (vor Therapieeinleitung, nach 3tägiger nCPAP-Therapie und unter Dauertherapie > 6 Monate) mittels Varianzanalyse untersucht. Die Homogenität der Varianzen wurde dabei mit dem Bartlett-Test überprüft.

In einem letzten Schritt wurde der Scheffé-Test, ein Posthoc-Test, zur Überprüfung aller möglichen linearen Kombinationen von Gruppenmittelwerten durchgeführt, d.h. dass Mittelwertsunterschiede sowohl zwischen den beiden Gruppen (Gesunde vs. OSAS-Patienten) als auch bzgl. des Therapieverlaufs für die globale sowie die bereichsspezifische Lebensqualität überprüft wurden.

Um eine Vergleichbarkeit der Effektivität der nCPAP-Therapie bzgl. der sehr unterschiedlichen Lebensqualitätsparameter zu ermöglichen, wurden in einem letzten Schritt die Effektstärken (ES) ermittelt. Zu ihrer Berechnung wurde die von Gerdes vorgeschlagene Formel ES $=\mu 1-\mu 2 / \sigma \times 1$ angewandt $(\mu 1=$ Mittelwert der 1 . Messung, $\mu 2=$ Mittelwert der 2 . Messung, $\sigma \times 1=$ Standardabweichung der 1 . Messung) $[8,9]$.

Nach Schuhmann u. Mitarb. (1997) und Hartung \& Herzog (1995) wurden die Effektstärken in gering $(<0,4)$, mittel $(0,4-0,8)$ und stark $(>0,8)$ eingeteilt $[8,9]$.
Tab.2 Mittelwerte der globalen Lebensqualität mittels der Satisfaction With Life Scale (SWLS)

\begin{tabular}{|c|c|c|c|c|}
\hline & \multirow[t]{2}{*}{ Gesunde } & \multicolumn{3}{|c|}{ OSAS-Patienten } \\
\hline & & vor Therapie & nach 3 Tagen & $\begin{array}{l}\text { nach > } 6 \\
\text { Monaten }\end{array}$ \\
\hline $\begin{array}{l}\text { Mittelwert } \pm \\
\text { Standard- } \\
\text { abweichung }\end{array}$ & $23,8 \pm 5,6$ & $23,6 \pm 6,6$ & $24,8 \pm 5,5$ & $25,2 \pm 5,2$ \\
\hline
\end{tabular}

Ergebnisse

Zunächst wurde die Stichprobe hinsichtlich ihrer klinischen und demographischen Parameter untersucht. Es ergab sich eine normalverteilte Stichprobe.

\section{Satisfaction With Life Scale (SWLS)}

Die Überprüfung (Varianzanalyse) der globalen Lebensqualität mittels Satisfaction With Life Scale (SWLS) ergab keinen signifikanten Unterschied zwischen den Gesunden und den OSASPatienten (Tab. 2).

\section{Münchner Lebensqualitäts Dimensionen Liste (MLDL) Varianzanalyse}

In der bereichspezifischen Lebensqualität wiesen mittels Varianzanalyse 2 (von 4) Subskalen (Physische Zufriedenheit $\mathrm{p}<0,001$; Psychische Zufriedenheit $\mathrm{p}=0,001$ ) der Münchner Lebensqualitäts-Dimensionen-Liste MLDL-Z einerseits signifikante Unterschiede zwischen Gesunden und OSAS-Patienten, andererseits im Therapieverlauf auf (vgl. Tab. 3). Die Soziale Zufriedenheit ergab noch einen tendenziellen Unterschied $(p=0,072)$.

\section{Scheffé-Test (s. Tab. 4)}

Bei den paarweisen Untersuchungen mit dem Scheffé-Test fanden sich in der Subskala Physische Zufriedenheit (s. Abb.1) signifikante Unterschiede zwischen Gesunden und unbehandelten Patienten sowie Patienten nach Therapieeinleitung. Des Weiteren ergaben sich signifikante Unterschiede zwischen den Patienten vor Therapie und unter nCPAP-Dauertherapie sowie zwischen Patienten nach Therapieeinleitung und unter Dauertherapie.

In der Psychischen Zufriedenheit (s. Abb. 2) fand sich ein signifikanter Unterschied zwischen den Gesunden und den Patienten

Tab.3 Mittelwerte der bereichsspezifischen Lebensqualität bei der Münchner Lebensqualitäts-Dimensionen-Liste (MLDL)

\begin{tabular}{|c|c|c|c|c|c|}
\hline & & \multirow[t]{2}{*}{ Gesunde } & \multicolumn{3}{|c|}{ OSAS-Patienten } \\
\hline & & & vor Therapie & nach 3 Tagen & nach > 6 Monaten \\
\hline Alltagsleben & Mittelwert \pm Standardabweichung & $36,3 \pm 3,0$ & $35,6 \pm 10,1$ & $38,1 \pm 9,3$ & $38,5 \pm 7,4$ \\
\hline physische Zufriedenheit & Mittelwert \pm Standardabweichung & $37,1 \pm 8,4$ & $29,2 \pm 8,8$ & $30,2 \pm 8,1$ & $35,4 \pm 7,8$ \\
\hline psychische Zufriedenheit & Mittelwert \pm Standardabweichung & $29,6 \pm 5,8$ & $23,6 \pm 8,6$ & $26,3 \pm 7,1$ & $28,4 \pm 6,6$ \\
\hline soziale Zufriedenheit & Mittelwert \pm Standardabweichung & $38,4 \pm 8,4$ & $35,3 \pm 12,8$ & $37,5 \pm 10,8$ & $41,0 \pm 6,8$ \\
\hline
\end{tabular}


Tab. 4 Scheffé-Test für die bereichsspezifische Lebensqualität bei der Münchner Lebensqualitäts-Dimensionen-Liste (MLDL)

\begin{tabular}{|c|c|c|c|}
\hline Subskala & Gruppe & & Signifikanz (p) \\
\hline \multirow[t]{6}{*}{ Alltagsleben } & Gesunde & OSAS-Patienten vor Therapie & ,997 \\
\hline & & OSAS-Patienten nach 3 Tagen & ,823 \\
\hline & & OSAS-Patienten nach > 6 Monaten & ,702 \\
\hline & OSAS-Patienten vor Therapie & OSAS-Patienten nach 3 Tagen & ,857 \\
\hline & & OSAS-Patienten nach > 6 Monaten & ,723 \\
\hline & OSAS-Patienten nach 3 Tagen & OSAS-Patienten nach > 6 Monaten & ,997 \\
\hline \multirow[t]{6}{*}{ physische Zufriedenheit } & Gesunde & OSAS-Patienten vor Therapie & $<, 001$ \\
\hline & & OSAS-Patienten nach 3 Tagen &, 005 \\
\hline & & OSAS-Patienten nach > 6 Monaten & ,854 \\
\hline & OSAS-Patienten vor Therapie & OSAS-Patienten nach 3 Tagen & ,984 \\
\hline & & OSAS-Patienten nach > 6 Monaten &, 005 \\
\hline & OSAS-Patienten nach 3 Tagen & OSAS-Patienten nach > 6 Monaten &, 051 \\
\hline \multirow[t]{6}{*}{ psychische Zufriedenheit } & Gesunde & OSAS-Patienten vor Therapie &, 002 \\
\hline & & OSAS-Patienten nach 3 Tagen &, 246 \\
\hline & & OSAS-Patienten nach > 6 Monaten & ,914 \\
\hline & OSAS-Patienten vor Therapie & OSAS-Patienten nach 3 Tagen & ,449 \\
\hline & & OSAS-Patienten nach > 6 Monaten &, 019 \\
\hline & OSAS-Patienten nach 3 Tagen & OSAS-Patienten nach > 6 Monaten & ,616 \\
\hline \multirow[t]{6}{*}{ soziale Zufriedenheit } & Gesunde & OSAS-Patienten vor Therapie & ,857 \\
\hline & & OSAS-Patienten nach 3 Tagen & ,981 \\
\hline & & OSAS-Patienten nach > 6 Monaten & ,708 \\
\hline & OSAS-Patienten vor Therapie & OSAS-Patienten nach 3 Tagen & ,984 \\
\hline & & OSAS-Patienten nach > 6 Monaten &, 167 \\
\hline & OSAS-Patienten nach 3 Tagen & OSAS-Patienten nach > 6 Monaten &, 461 \\
\hline
\end{tabular}

vor Therapie sowie einer zwischen den unbehandelten Patienten und denen unter Dauertherapie.

\section{Short Form-36 des Health Survey (SF-36) Varianzanalyse}

Bei der Short Form-36 des Health Survey SF-36 ergaben 7 (von 8) Subskalen signifikante bis höchst signifikante Unterschiede (Ausnahme: Emotionale Rollenfunktion; vgl. Tab. 5).

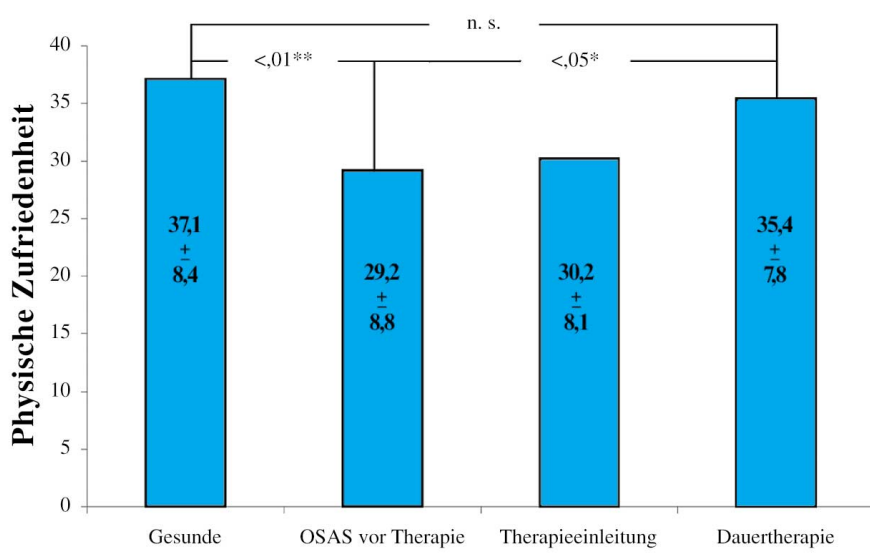

Diagnose

Abb. 1 Mittelwerte der bereichsspezifischen Lebensqualität (MLDL: Physische Zufriedenheit). Gesunde Personen $(n=40)$ und OSAS-Patienten $(n=41$; a: vor Therapieeinleitung, b: nach 3tägiger nCPAP-Therapie, : nach > 6 Monaten nCPAP).
Die Ergebnisse der Subskalen des SF-36 (s. Tab. 6) weisen höchst signifikante Unterschiede (Soziale Funktionen, Vitalität und Körperliche Funktionen), hoch signifikante Unterschiede (Allgemeine Gesundheitswahrnehmung, Schmerz und Körperliche Rollenfunktion) sowie signifikante Unterschiede (Psychisches Wohlbefinden) einerseits zwischen Gesunden und OSAS-Patienten, andererseits im Therapieverlauf auf. Bei der Subskala Emotionale Rollenfunktion zeigten sich keine signifikanten Unterschiede.

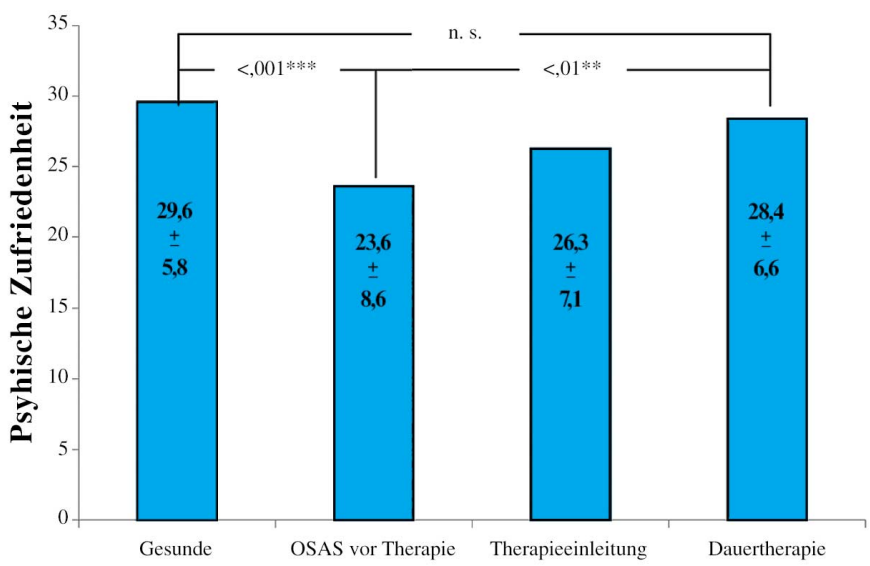

Diagnose

Abb. 2 Mittelwerte der bereichsspezifischen Lebensqualität (MLDL: Psychische Zufriedenheit). Gesunde Personen $(n=40)$ und OSAS-Patienten ( $n=41$; a: vor Therapieeinleitung, b: nach 3tägiger nCPAP-Therapie, $c$ : nach > 6 Monaten $\mathrm{nCPAP}$ ). 
Tab.5 Mittelwerte der bereichsspezifischen Lebensqualität bei der Short Form-36 des Health Survey (SF-36)

\begin{tabular}{|c|c|c|c|c|c|}
\hline & & \multirow[t]{2}{*}{ Gesunde } & \multicolumn{3}{|c|}{ OSAS-Patienten } \\
\hline & & & vor Therapie & nach 3 Tagen & nach > 6 Monaten \\
\hline allgm. Gesundh.-wahrnehmg. & Mittelwert \pm Standardabweichung & $41,6 \pm 11,6$ & $43,7 \pm 12,7$ & $46,3 \pm 8,4$ & $36,9 \pm 12,2$ \\
\hline emotionale Rollenfunktion & Mittelwert \pm Standardabweichung & $85,8 \pm 26,0$ & $67,5 \pm 42,2$ & $69,2 \pm 39,5$ & $75,0 \pm 36,8$ \\
\hline körperliche Funktionen & Mittelwert \pm Standardabweichung & $87,8 \pm 19,2$ & $65,8 \pm 26,5$ & $69,9 \pm 28,0$ & $72,9 \pm 23,4$ \\
\hline körperliche Rollenfunktion & Mittelwert \pm Standardabweichung & $76,8 \pm 32,7$ & $51,2 \pm 39,9$ & $49,4 \pm 42,0$ & $64,9 \pm 43,8$ \\
\hline psychisches Wohlbefinden & Mittelwert \pm Standardabweichung & $70,5 \pm 13,2$ & $60,2 \pm 21,3$ & $62,1 \pm 19,9$ & $69,4 \pm 18,2$ \\
\hline Schmerzen & Mittelwert \pm Standardabweichung & $84,0 \pm 22,9$ & $61,6 \pm 28,2$ & $65,3 \pm 28,6$ & $67,8 \pm 29,9$ \\
\hline soziale Funktionen & Mittelwert \pm Standardabweichung & $86,2 \pm 17,2$ & $65,5 \pm 29,6$ & $63,4 \pm 26,7$ & $77,4 \pm 22,3$ \\
\hline Vitalität & Mittelwert \pm Standardabweichung & $60,8 \pm 18,0$ & $40,8 \pm 20,2$ & $44,5 \pm 21,6$ & $59,6 \pm 18,8$ \\
\hline
\end{tabular}

\section{Scheffé-Test (s. Tab. 7)}

Bei den paarweisen Untersuchungen mit dem Scheffé-Test fanden sich in den Subskalen Körperliche Funktionen, Soziale Funktionen und Vitalität die signifikantesten Unterschiede zwischen den Gesunden und den Patienten vor Therapie bzw. nach Therapieeinleitung.

Bei der Subskala Vitalität (s. Abb.3) ergaben sich außerdem ebenfalls signifikante Unterschiede zwischen den Patienten vor Therapiebeginn bzw. nach Therapieeinleitung und denen unter Dauertherapie.

Zwischen den Gesunden und den Patienten unter nCPAP-Dauertherapie zeigten sich in den Subskalen Soziale Funktionen und Vitalität keine signifikanten Unterschiede mehr. schieden, an. Die schlechteste Lebensqualität ergab sich bei den untherapierten Patienten.

\section{Effektstärken der Skalen}

Der Vergleich der Effektstärken (s. Tab. 8) ergab nach einer 3tägigen nCPAP-Therapie nur sehr geringfügige Effekte für die verschiedenen Lebensqualitätsdomänen. Der beste Effekt zeigte sich bei der Psychischen Zufriedenheit der MLDL (ES = 0,31).

Bei der Überprüfung der Lebensqualität unter einer $>6$ monatigen nCPAP-Therapie ergaben sich bessere Effektstärken: Der höchste Effekt zeigte sich bei der Skala Vitalität im SF-36 nach $>6$ Monaten $(E S=0,93)$. Gute Effektstärken fanden sich in den Domänen Physische Zufriedenheit ( $E S=0,70$ ) und Psychische Zufriedenheit der MLDL (ES = 0,56).

\section{Diskussion}

Die Annahme, dass sich OSAS-Patienten bezüglich der Lebensqualitäts-Parameter von Gesunden deutlich unterscheiden, konnte bestätigt werden. Bestätigt wurde außerdem der Einfluss der obstruktiven Schlafapnoe auf das Tageserleben und die subte), deren Werte sich in den einzelnen Skalen unwes (keine signifikanten Unterschiede) von den Gesunden unter-

Tab.6 Varianzanalyse bei der bereichsspezifischen Lebensqualität bei der Short Form-36 des Health Survey (SF-36)

\begin{tabular}{llc}
\hline & F-Wert & Signifikanz $(\boldsymbol{p})$ \\
\hline allgm. Gesundh.-wahrnehmg. & 5,179 &, 002 \\
\hline emotionale Rollenfunktion & 2,035 &, 111 \\
\hline körperliche Funktionen & 6,098 & $<, 001$ \\
körperliche Rollenfunktion & 4,211 &, 007 \\
psychisches Wohlbefinden & 3,198 &, 025 \\
Schmerzen & 5,196 &, 002 \\
\hline soziale Funktionen & 7,756 & $<, 001$ \\
Vitalität & 11,053 & $<, 001$ \\
\hline
\end{tabular}

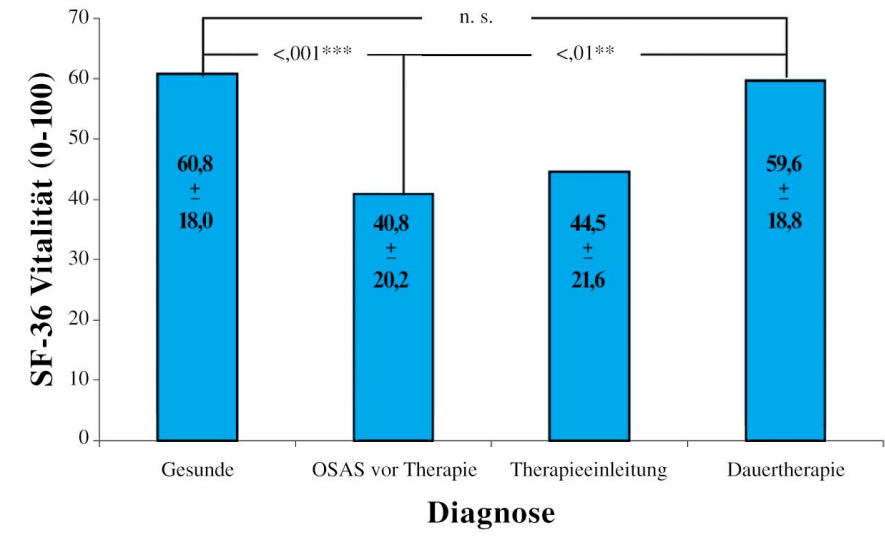

Abb. 3 Mittelwerte der bereichsspezifischen Lebensqualität (SF-36: Vitalität). Gesunde Personen $(n=40)$ und OSAS-Patienten $(n=41$; a: vor Therapieeinleitung, b: nach 3tägiger nCPAP-Therapie, c: nach > 6 Monaten nCPAP). 
Tab.7 Scheffé-Test für die bereichsspezifische Lebensqualität bei der Short Form-36 des Health Survey (SF-36)

\begin{tabular}{|c|c|c|c|}
\hline Subskala & Gruppe & & Signifikanz (p) \\
\hline \multirow[t]{6}{*}{ allgm. Gesundh.-wahrnehmg. } & Gesunde & OSAS-Patienten vor Therapie &, 877 \\
\hline & & OSAS-Patienten nach 3 Tagen & ,320 \\
\hline & & OSAS-Patienten nach > 6 Monaten & ,316 \\
\hline & OSAS-Patienten vor Therapie & OSAS-Patienten nach 3 Tagen & ,771 \\
\hline & & OSAS-Patienten nach > 6 Monaten &, 062 \\
\hline & OSAS-Patienten nach 3 Tagen & OSAS-Patienten nach > 6 Monaten &, 003 \\
\hline \multirow[t]{6}{*}{ emotionale Rollenfunktion } & Gesunde & OSAS-Patienten vor Therapie &, 181 \\
\hline & & OSAS-Patienten nach 3 Tagen & ,251 \\
\hline & & OSAS-Patienten nach > 6 Monaten &, 627 \\
\hline & OSAS-Patienten vor Therapie & OSAS-Patienten nach 3 Tagen & ,998 \\
\hline & & OSAS-Patienten nach > 6 Monaten &, 844 \\
\hline & OSAS-Patienten nach 3 Tagen & OSAS-Patienten nach > 6 Monaten & ,917 \\
\hline \multirow[t]{6}{*}{ körperliche Funktionen } & Gesunde & OSAS-Patienten vor Therapie &, 002 \\
\hline & & OSAS-Patienten nach 3 Tagen &, 015 \\
\hline & & OSAS-Patienten nach > 6 Monaten &, 061 \\
\hline & OSAS-Patienten vor Therapie & OSAS-Patienten nach 3 Tagen & ,907 \\
\hline & & OSAS-Patienten nach > 6 Monaten &, 637 \\
\hline & OSAS-Patienten nach 3 Tagen & OSAS-Patienten nach > 6 Monaten & ,958 \\
\hline \multirow[t]{6}{*}{ körperliche Rollenfunktion } & Gesunde & OSAS-Patienten vor Therapie &, 042 \\
\hline & & OSAS-Patienten nach 3 Tagen &, 025 \\
\hline & & OSAS-Patienten nach > 6 Monaten &, 605 \\
\hline & OSAS-Patienten vor Therapie & OSAS-Patienten nach 3 Tagen & ,998 \\
\hline & & OSAS-Patienten nach > 6 Monaten & ,490 \\
\hline & OSAS-Patienten nach 3 Tagen & OSAS-Patienten nach > 6 Monaten & ,376 \\
\hline \multirow[t]{6}{*}{ psychisches Wohlbefinden } & Gesunde & OSAS-Patienten vor Therapie &, 101 \\
\hline & & OSAS-Patienten nach 3 Tagen & ,249 \\
\hline & & OSAS-Patienten nach > 6 Monaten & ,995 \\
\hline & OSAS-Patienten vor Therapie & OSAS-Patienten nach 3 Tagen & ,973 \\
\hline & & OSAS-Patienten nach > 6 Monaten &, 162 \\
\hline & OSAS-Patienten nach 3 Tagen & OSAS-Patienten nach > 6 Monaten & ,360 \\
\hline \multirow[t]{6}{*}{ Schmerzen } & Gesunde & OSAS-Patienten vor Therapie &, 005 \\
\hline & & OSAS-Patienten nach 3 Tagen &, 028 \\
\hline & & OSAS-Patienten nach > 6 Monaten & ,073 \\
\hline & OSAS-Patienten vor Therapie & OSAS-Patienten nach 3 Tagen & ,946 \\
\hline & & OSAS-Patienten nach > 6 Monaten & ,788 \\
\hline & OSAS-Patienten nach 3 Tagen & OSAS-Patienten nach > 6 Monaten & ,982 \\
\hline \multirow[t]{6}{*}{ soziale Funktionen } & Gesunde & OSAS-Patienten vor Therapie &, 003 \\
\hline & & OSAS-Patienten nach 3 Tagen & ,001 \\
\hline & & OSAS-Patienten nach > 6 Monaten &, 442 \\
\hline & OSAS-Patienten vor Therapie & OSAS-Patienten nach 3 Tagen & ,984 \\
\hline & & OSAS-Patienten nach > 6 Monaten &, 186 \\
\hline & OSAS-Patienten nach 3 Tagen & OSAS-Patienten nach > 6 Monaten &, 083 \\
\hline \multirow[t]{6}{*}{ Vitalität } & Gesunde & OSAS-Patienten vor Therapie & $<, 001$ \\
\hline & & OSAS-Patienten nach 3 Tagen &, 004 \\
\hline & & OSAS-Patienten nach > 6 Monaten & ,996 \\
\hline & OSAS-Patienten vor Therapie & OSAS-Patienten nach 3 Tagen &, 871 \\
\hline & & OSAS-Patienten nach > 6 Monaten & $<, 001$ \\
\hline & OSAS-Patienten nach 3 Tagen & OSAS-Patienten nach > 6 Monaten & ,008 \\
\hline
\end{tabular}

jektiv erlebte Lebensqualität sowie deren Verbesserung unter einer nCPAP-Therapie.

So fanden sich signifikante Unterschiede in der bereichsspezifischen Lebensqualität, nicht aber in der globalen. Dieses Ergebnis könnte als Beleg für die Multidimensionalität der Lebensqualität gewertet werden. Außerdem ergab sich ein signifikanter Zusam- menhang hinsichtlich der Therapielänge. So unterschieden sich Gesunde und Patienten unter Dauertherapie nur noch unwesentlich in den einzelnen Subskalen der MLDL und der SF-36.

Die Ergebnisse bei der MLDL und bei der SF-36 ergaben teilweise starke Unterschiede in der bereichsspezifischen Lebensqualität zwischen Gesunden und OSAS-Patienten einerseits und im The- 
Tab.8 Effektstärken der Lebensqualitätsparameter bei 41 OSASPatienten unter nCPAP-Therapie

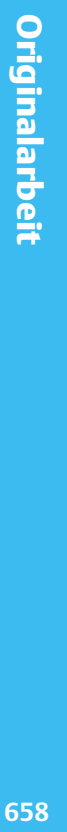

\begin{tabular}{|llll|}
\hline & & Effektstärke & \\
\hline \multirow{2}{*}{ SWLS } & & nach 3 Tagen & nach > 6 Monaten \\
\hline MLDL & Alltagsleben & 0,18 & 0,24 \\
& physische Zufriedenheit & 0,25 & 0,29 \\
\hline & psychische Zufriedenheit & 0,11 & 0,70 \\
\hline \multirow{2}{*}{ SF-36 } & soziale Zufriedenheit & 0,17 & 0,56 \\
& allgm. Gesundh.-wahrnehmg. & 0,20 & 0,44 \\
& emotionale Rollenfunktion & 0,04 & 0,54 \\
& körperliche Funktionen & 0,15 & 0,18 \\
\hline & körperliche Rollenfunktion & 0,04 & 0,27 \\
\hline & psychisches Wohlbefinden & 0,09 & 0,34 \\
\hline & Schmerzen & 0,13 & 0,43 \\
\hline & soziale Funktionen & 0,07 & 0,40 \\
\hline & Vitalität & 0,18 & 0,93 \\
\hline
\end{tabular}

rapieverlauf andererseits. So zeigten sich in verschiedenen Subskalen der MLDL und der SF-36 deutliche Unterschiede zwischen den Gesunden und den unbehandelten Patienten sowie denen nach Therapieeinleitung.

Bestätigt wurde dadurch sowohl der Einfluss der obstruktiven Schlafapnoe auf die subjektiv erlebte Lebensqualität als auch deren Verbesserung unter nCPAP-Therapie. Des weiteren ergab sich ein signifikanter Effekt hinsichtlich der Therapielänge. Dieser Befund deckt sich auch mit den Ergebnissen von Trumm u. Mitarb. [21]), Bolitschek u. Mitarb. [1]) und D'Ambrosio u. Mitarb. [7]), die feststellten, dass OSAS die Lebensqualität erheblich einschränkt und dass es unter nCPAP-Therapie zu einer deutlichen Verbesserung kommt.

In den Lebensqualitätsbereichen Zufriedenheit im alltäglichen Leben, Soziale Zufriedenheit (MLDL) und Emotionale Rollenfunktion (SF-36) scheinen bei Patienten mit obstruktiver Schlafapnoe weniger Beeinträchtigungen aufzutreten, da sie sich in diesen Bereichen nicht von den Gesunden unterscheiden.

Bei der Physischen Zufriedenheit und der Psychischen Zufriedenheit (MLDL) unterschieden sich die unbehandelten Patienten und die unter Therapieeinleitung sehr deutlich von den Gesunden. Unter Dauertherapie nahm der Score in diesen Lebensqualitäts-Domänen deutlich zu. In den Untersuchungen von [1] fanden sich sogar in allen vier Domänen der MLDL relevante Unterschiede zwischen unbehandelten OSAS-Patienten und gesunden Kontrollen. Unter einer $>3$ monatigen nCPAP-Therapie stieg die Lebensqualität dieser Patienten in allen vier Lebensqualitätsbereichen so an, dass sich zwischen den Patienten und den Kontrollen keine Unterschiede mehr fanden [1].

Auch in verschiedenen Lebensqualitäts-Bereichen der SF-36 fanden sich Unterschiede zwischen Gesunden, teilweise auch zwischen den OSAS-Patienten unter nCPAP-Dauertherapie und den unbehandelten Patienten bzw. denen nach Therapieeinleitung, u. a. bei der Allgemeinen Gesundheitswahrnehmung, den Körperlichen Funktionen, dem Schmerz, den Sozialen Funktionen und der Vitalität. Die Unterschiede in diesen Lebensqualitäts-Bereichen scheinen besonders auf eine unterschiedliche Lebensqualitäts-Wahrnehmung der Gesunden und der schlafgestörten Patienten hinzuweisen.

Zu ähnlichen Ergebnissen kamen auch D’Ambrosio u. Mitarb. in allen Domänen der SF-36 ergaben sich relevante Unterschiede zwischen unbehandelten OSAS-Patienten und gesunden Kontrollpersonen. Unter einer 8-wöchigen nCPAP-Therapie stieg die Lebensqualität dieser Patienten in Bereichen Vitalität, Soziale Funktionen und Psychisches Wohlbefinden bis in den Normalbereich an [7]. Problematisch bleibt in den vorrausgegangenen Studien allerdings die Stichprobenzusammensetzung mit sehr unterschiedlichen Gruppengrößen und unterschiedlichen Komorbiditäten.

Die Berechnung der Effektstärken über die Effektivität therapeutischer Maßnahmen in den verschiedenen Lebensqualitätsdomänen zeigt, dass die Skala Vitalität der SF-36 und die Skalen Physische und Psychische Zufriedenheit der MLDL die besten Parameter zur Beschreibung der unter längerfristiger nCPAP-Therapie verbesserten Lebensqualität darstellen. Eine kurzfristige Erfassung der Lebensqualität ( 3 Tage nCPAP) erscheint hingegen wenig sinnvoll.

In diesem Zusammenhang soll auch kurz auf die Praktikabilität der verschiedenen Lebensqualitäts-Fragebögen eingegangen werden. Während die SF-36 sehr aufwendig (Testzeit: 16,88 +/-6,62 min. und Auswertung: ca. 20-25 min.) und schwierig (Verständlichkeit: z.B. doppelte Verneinungen) ist, ist die MLDL (Testzeit: 6,05+/-2,54 min. und Auswertung: ca. 5 min.) zeitökonomisch und anwenderfreundlich [4].

Für die praktische Umsetzung in die klinische Anwendung des MLDL-Fragebogens ergibt sich folgende Konsequenz: Wird bei Kontroll-Untersuchungen unter CPAP in diesen beiden Domänen der Normalwert nicht erreicht, sollten die Therapie bzw. die Komorbiditäten nochmals kritisch überprüft werden.

Schlussfolgernd lässt sich sagen, dass die Fragen zur Vitalität in dem SF-36-Fragebogen die Besserung unter CPAP am besten beschreiben. Aber in der klinischen Routine stellt die MLDL mit ihren 2 Domänen Physische und Psychische Zufriedenheit aufgrund ihrer einfachen Durchführung und Auswertung im Vergleich zur SF-36 das geeignetere Instrument zur Erfassung von verbesserter Lebensqualität durch eine nCPAP-Therapie dar.

\section{Literatur}

${ }^{1}$ Bolitschek J et al. Impact of nasal continuous positive airway pressure treatment on quality of life in patients with obstructive sleep apnea. Eur Respir J 1998; 11 (4): 890-894

2 Büttner A. Schlafqualität, gesundheitsbezogene Kontrollüberzeugungen und die Lebensqualität von Narkolepsie-, Insomnie- und OSAS-Patienten. Universität Marburg: Diplomarbeit im Fachbereich Psychologie, 1997

3 Büttner A, Rühle K-H. Lebensqualität bei obstruktiver Schlafapnoe vor und unter nCPAP. Pneumologie 2002a ; 56: 49

${ }^{4}$ Büttner A, Rühle K-H. Lebensqualität vor und nach nCPAP - Ein Vergleich mittels MLDL, SF-36, FOSQ und SAQLI. Somnologie 2002b ; 6 (Suppl.1): 31 
${ }^{5}$ Bullinger M, Ludwig M, Steinbüchel N v.. Lebensqualität bei kardiovaskulären Erkrankungen. Göttingen: Hogrefe-Verlag für Psychologie, 1991

${ }^{6}$ Bullinger M, Kirchberger I, Ware J. Der deutsche SF-36 Health Survey. Zeitschrift für Medizinische Psychologie 1995; 3 (1): 21 - 36

${ }^{7}$ DaposAmbrosio C et al. Quality of life in patients with obstructive sleep apnea. Effect of nasal continuous positive airway pressure - A prospective study. Chest 1999; 115 (1): 123 - 129

8 Fischer J, Raschke F. Zielorientierte Messung von kurz-, mittel- und langfristigen Effekten in der pneumologischen Rehabilitation. Pneumologie 2000; 54: 458-463

${ }^{9}$ Gerdes N. Methodik. In: Gerdes N, Weidmann H, Jäckel WH (Hrsg). Die Protos-Studie - Ergebnisqualität stationärer Rehabilitation in 15 Wittgensteiner Kliniken. Darmstadt: Steinkopf-Verlag, 2000: 22 - 25

10 Görtelmeyer R. On the Development of a Standardized Sleep Inventory for the Assessment of Sleep. In: Kubicki St, Herrmann WM (Hrsg). Methods of Sleep Research. New York: G. Fischer Verlag, 1985

${ }^{11}$ Görtelmeyer R. Typologie des Schlafverhaltens - Eine empirische Untersuchung an berufstätigen Personen. In: Wittchen H-U (Hrsg). Beiträge zur klinischen Psychologie und Psychotherapie, Bd.4. Regensburg: S. Roderer Verlag, 1988

12 Langer B, Schulz H. Bessert sich die Befindlichkeit von Patienten mit Schlaf-Apnoe-Syndrom unter einer nCPAP-Therapie? Somnologie 1998; 2 (Suppl. 1): 16

${ }^{13}$ Meier-Ewert K. Differenzialdiagnose abnormer Tagesschläfrigkeit. Schweizerische Rundschau für Medizin 1988; 35: 920-925

${ }^{14}$ Meier-Ewert K. Abnorme Tagesschläfrigkeit - Ansätze einer Typologie. Therapeutische Umschau 1991; 48: 746-750

${ }^{15}$ Rupprecht R. Lebensqualität - Theoretische Konzepte und Ansätze zur Operationalisierung. Dissertation der Philosophischen Falkutät I, F.Alexander-Universität Erlangen-Nürnberg, 1993

${ }^{16}$ Sanner BM, Klewer J, Trumm A et al. Long-term treatment with continuous positive airway pressure improves quality of life in obstructive sleep apnea syndrome. Eur Respir J 2000; 16 (1): 118-122

17 Sanner BM, Tepel M, Esser M et al. Sleep-related breathing disorders impair quality of life in haemodialysis recipients. Nephrol Dial Transplant 2002; 17 (7): $1260-1265$

${ }^{18}$ Schmidt RF. Integrative Funktionen des Zentralnervensystems. In: Schmidt RF, Thews G (Hrsg). Physiologie des Menschen, 22. Aufl. Berlin, Heidelberg, New York, Tokio: Springer Verlag, 1985

${ }^{19}$ Schwenkhagen U et al. Die Bedeutung des Konzepts der Lebenszufriedenheit - Ein Vergleich guter und schlechter Schläfer. In: Becker-Carus Ch (Hrsg). Fortschritte der Schlafmedizin - Aktuelle Beiträge zur Insomnie-Forschung. Münster: Lit. Verlag, 1995

${ }^{20}$ Shneerson JM, Smith IE. In the SF-36 sensitive to sleep disruption? - A study in subjects with sleep apnoea. J. Sleep Res. 1995; 4: 183-188

${ }^{21}$ Trumm A, Sanner B, Klewer J, Burmann-Urbanek M et al. Der Einfluss einer Langzeit-nCPAP-Therapie auf die Lebensqualität von Patienten mit obstruktiver Schlafapnoe. Somnologie 1998; 2 (Suppl. 1): 16

22 Westhoff G. Handbuch psychosozialer Messinstrumente. Göttingen, Berlin, Heidelberg, New York: Springer Verlag, 1993

${ }^{23}$ Zeitlhofer J. Schlafstörungen und Lebensqualität - Epidemiologische Daten aus Österreich. Somnologie 1998; 2 ( Suppl. 1): 6

\section{Stipendium}

Ausschreibung von Promotionsstipendien der Deutschen

Gesellschaft für Pneumologie

\section{- Deadline für die Abgabe der Anträge: 31. Dezember 2004 -}

Die Deutsche Gesellschaft für Pneumologie (DGP) schreibt 2004 erstmals Promotionsstipendien aus. Die Stipendien dienen der Förderung des wissenschaftlichen Nachwuchses in der Pneumologie; sie sind mit jeweils 40000 Euro dotiert.

Die Bewerber sollen nicht über 40 Jahre alt sein, sie müssen ihre Arbeitsstätte in Deutschland haben. Die Anträge sollen entsprechend den Vorgaben eines DFG-Antrags („Einzelförderung im Normalverfahren“) abgefasst sein. Entscheidend für die Vergabe der Stipendien ist die klinisch-wissenschaftliche Qualität der Anträge, über die in einem externen Begutachtungsverfahren entschieden wird.

Die Bewerber werden gebeten, ihre Arbeit bis zum 31. Dezember 2004 in 8-facher Ausfertigung an den Geschäftsführer der Gesellschaft, Prof. Dr. Adrian Gillissen, Robert-Koch-Klinik, NikolaiRumjanzew-Straße 100, 04207 Leipzig, zu schicken.

Prof. Dr. Helgo Magnussen (Präsident) Prof. Dr. Adrian Gillissen (Geschäftsführer) 